\title{
Is Cytology Safest Modality Available in Armamentarium for Diagnosis of Hodgkin's Lymphoma in Pregnancy?
}

\author{
Richa Bhartiya ${ }^{1}$, Pallavi Agrawal ${ }^{2 *}$, Kumar Rajnish ${ }^{2}$ and Nawanita Kumari ${ }^{2}$ \\ ${ }^{1}$ Department of Pathology, Grant Government Medical College, Mumbai (Maharashtra) India \\ ${ }^{2}$ Department of Pathology, Patna Medical College Hospital (PMCH), Ashok Rajpath, Patna (Bihar) India
}

\begin{abstract}
Hodgkin's lymphoma (HL) is one of the curable diseases in pregnancy. Since pregnant females are immuno-compromised, so it is important to know about the course and progression of this common entity in pregnancy. Fine needle aspiration cytology is minimally invasive and a safe approach to diagnose and subtype such cases as early diagnosis and effective treatment will lead to better outcome of the mother and fetus. Here we present a case of nodular sclerosis Hodgkin's lymphoma in a pregnant female diagnosed on cytology with histopathological confirmation and immuno-histochemical analysis.
\end{abstract}

\section{Keywords: Fine Needle Aspiration, Cytology, Lymph Node, Lymphoma}

\section{Introduction}

Hodgkin's lymphoma (HL) is one of the most curable forms of lymphoma. It is potentially curable even in late stages. Although hodgkin's lymphoma is commonly seen in reproductive age group, the reported incidence of HL in pregnant females is as low as $3.2 \% \cdot{ }^{[1]}$ Fine needle aspiration cytology proves to be an effective minimally invasive tool in early diagnosis and treatment of this disease especially in pregnant females. Herein we present a rare case of Hodgkin's lymphoma in pregnancy and the role of FNAC in early diagnosis and treatment in these rare presentations.

\section{Case Report}

A 35-year-old multiparous woman at 22 weeks of pregnancy presented with bilateral axillary lymphadenopathy for past 20 days. She also had fever for last 5 days. She had no complaints of night sweats but had a past history of taking anti-tubercular drugs (ATT) for 9 months, two years back. At that time also she had small axillary swellings which subsided after taking ATT. Now when she again developed similar swellings she started taking ATT on her own for 15 days. Since the swellings didn't subsided this time she visited the hospital for further evaluation. On clinical examination the left axillary lymph node was enlarged measuring $6 \times 5 \times 2 \mathrm{~cm}$, with soft rubbery consistency (Fig 1). The right axillary node was also soft and enlarged measuring $2 \times 2 \times 1 \mathrm{~cm}$. There was no other lymphadenopathy. She was advised for FNAC with the clinical suspicion of lymphoma and tuberculosis. Routine hematological investigations revealed haemoglobin of $12.5 \mathrm{gm} / \mathrm{dl}$, total leucocyte count of 10500 cells $/ \mathrm{mm}^{3}$ and platelet count of $296 \times 10^{9} / \mathrm{L}$. The erythrocyte sedimentation rate was $30 \mathrm{~mm} / \mathrm{hr}$. The iron profile was within normal limits. FNAC was performed from the bilateral axillary lymph nodes. Smears examined were cellular and show many mononuclear cells with prominent nucleoli. Focally large atypical bizarre cells having RS cell like morphology were also seen. The background shows polymorphous population of cells comprising of polymorphs, mature reactive lymphocytes, eosinophils and plasma cells. Admixed with these few granulomas were also identified (Fig $2 \& 3$ ). No caseation necrosis and langhan type of giant cell seen. Stain for acid-fast bacilli was negative. Finally, the possibility of Hodgkin's lymphoma was considered. A lymph node biopsy was performed under local anesthesia. Grossly a single large lymph node was received measuring $2 \times 2 \times 1 \mathrm{~cm}$. On microscopic examination the nodal architecture was distorted with band of sclerosis. The preserved areas revealed many atypical mononuclear cells with few classical RS cells. The background was polymorphous comprising of neutrophils, mature reactive lymphocytes, eosinophils and plasma cells (Fig 4 \& 5). On immuno-histochemistry these RS cells and mononuclear cells were positive for $\mathrm{CD}_{30}, \mathrm{CD}_{15}, \mathrm{PAX}-5$ (Fig 6 \& 7) and negative for ALK-1 thus ruling out the possibility of anaplastic large cell lymphoma. The proliferation index (Ki-67) was 60\%. Based on the morphology and immunohistochemistry possibility of nodular sclerosis Hodgkin's lymphoma was considered. Since the patient was pregnant she underwent only abdominal ultrasound where there were multiple enlarged para-aortic lymph nodes. Patient didn't give consent for bone marrow biopsy with the fear of spontaneous labor. Based on all the investigations done she was finally diagnosed as nodular sclerosis HL clinical 
stage IIs-A according to Ann Arbor staging classification. The patient underwent chemotherapy with doxorubicin, bleomycin, vinblastine, and dacarbazine (ABVD) for 6 cycles, every 4 weeks. The patient's pregnancy was monitored with regular ultrasounds. Till the subsequent two months when this case report was written the patient had an uneventful clinical course with absolutely normal fetus without any adverse effects of these drugs. Moreover, the axillary lymphadenopathy was gradually subsiding and the patient was feeling better.

Table 1: Clinicopathological features and immunohistochemical pattern in T cell rich, large B cell lymphoma (TCRBCL), lymphocyte predominant Hodgkin's lymphoma (LP-HL), and classical Hodgkin's lymphoma (CHL).

\begin{tabular}{|c|c|c|c|}
\hline Diagnostic criteria & TCRBCL & LP-HL & CHL \\
\hline \multicolumn{4}{|l|}{ Neoplastic } \\
\hline L\&H cells & $-1+$ & $+/-$ & - \\
\hline RS cells & $-1+$ & $-1+$ & - \\
\hline CD45 expression & + & + & - \\
\hline CD30 expression & - & - & + \\
\hline CD15 expression & - & - & $+/-$ \\
\hline CD20 expression & + & + & $-1+$ \\
\hline CD3 expression & - & - & $-/+$ \\
\hline EMA expression & $+1-$ & $+1-$ & Rare \\
\hline Ki-67 expression & High & High & High \\
\hline EBV & $-1+$ & $-1+$ & + Variable \\
\hline PAX5 & - & + & + \\
\hline \multicolumn{4}{|c|}{ Reactive component } \\
\hline $\mathrm{T}$ cells & Numerous & Moderate & Variable \\
\hline Bcl-6+/CD57+ rosettes & - & Numerous & - \\
\hline $\begin{array}{l}\text { Amount of CD20+ small } \\
\text { lymphocytes }\end{array}$ & Low & High & Low \\
\hline Histiocytes & Variable & Some & Variable \\
\hline Follicular dendritic cells & - & + & + \\
\hline \multicolumn{4}{|c|}{ Clinical findings } \\
\hline Stage & III/IV & $\mathrm{I} / \mathrm{II}$ & $\mathrm{I} / \mathrm{III}$ \\
\hline Bone marrow involvement & $+/-$ & - & - \\
\hline $\begin{array}{l}\text { Orderly progression in the } \\
\text { spread }\end{array}$ & - & - & + \\
\hline
\end{tabular}

Note: EMA [Epithelial Membrane Antigen], L/H [Lymphocytic/Histiocytic] (popcorn); RS [Reed Sternberg]. Popcorn cells show nuclei resembling those of centroblasts, with a polylobular profile, finely dispersed chromatin, and small nucleoli, which are often adjacent to the nuclear membrane

Table 2: Comparison of morphology and immunohistochemical pattern in anaplastic large cell lymphoma Hodgkin's-like (ALCL-HL) and classic Hodgkin's lymphoma (HL).

\begin{tabular}{|c|c|c|}
\hline & ALCL-HL & HL \\
\hline \multicolumn{2}{|c|}{ Morphological Features } \\
\hline Neoplastic component & Usually cohesive & Usually dispersed \\
\hline Reactive component & Often minor & Usually prevalent \\
\hline Reed-Sternberg cells & May be present & Always present \\
\hline Intrasinusoidal diffusion & Typical & Exceptional \\
\hline
\end{tabular}




\begin{tabular}{|c|c|c|}
\hline & ALCL-HL & HL \\
\hline & Immunophenotyping & + \\
\hline CD30 & + & $+/-$ \\
\hline CD45 & $-/+$ & $+/-$ \\
\hline CD15 & $+/-$ & + \\
\hline EMA & $-/+$ & + or $+/-$ \\
\hline P53 & $-/+$ & + \\
\hline EBV & - & - \\
\hline PAX-5 & + & + \\
\hline
\end{tabular}

EBV-Epstein-Barr virus

EMA - Epithelial Membrane Antigen

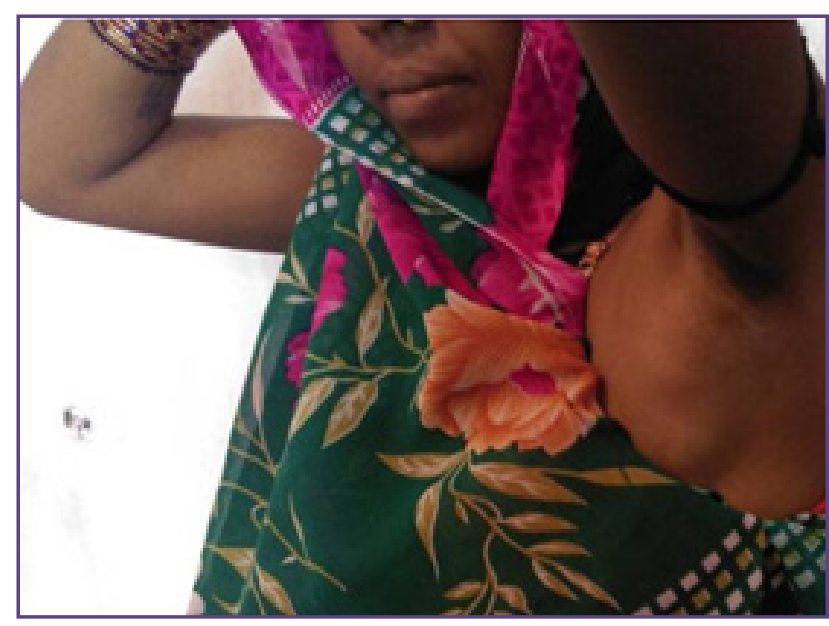

Fig. 1: Clinical image showing axillary swelling of left side.

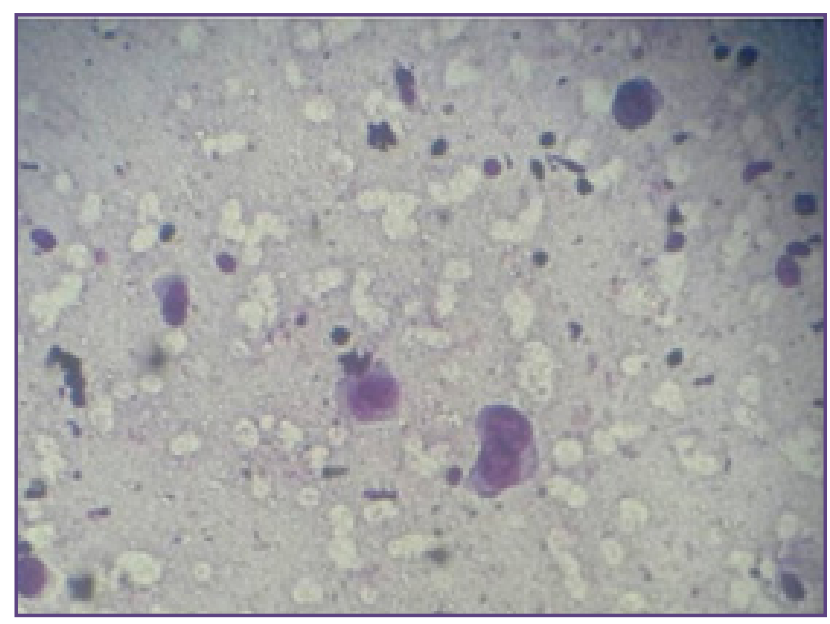

Fig. 3: Cytosmear showing classical Reed Sternberg cell as a large binucleate cell with each nucleus forming a mirror image of the other. Nuclei are vesicular and each has a very prominent eosinophilic central nucleolus. (Giemsa x 40X).

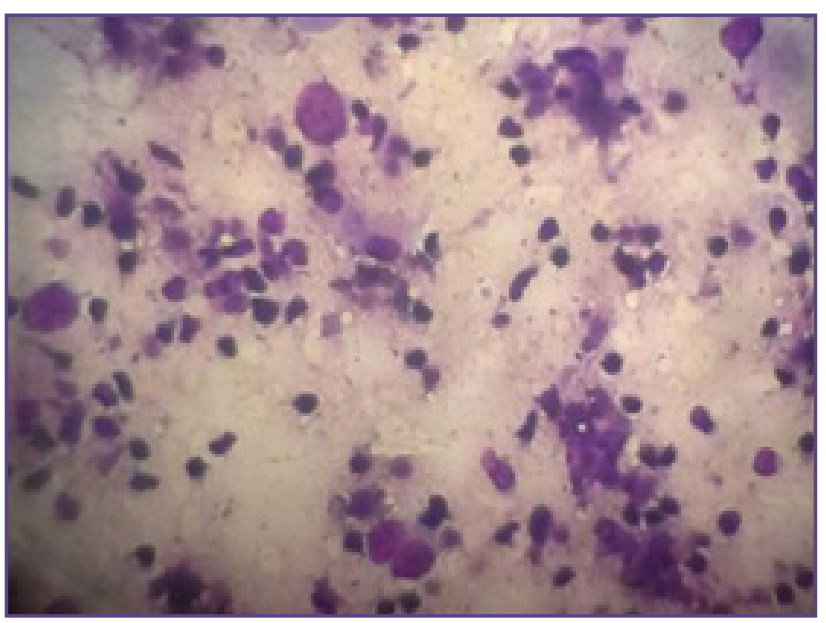

Fig 2 Cytosmear showing a typical diagnostic Reed Sternberg (RS) cell with in a compositeinflammatory milieu. (Giemsax 10X).

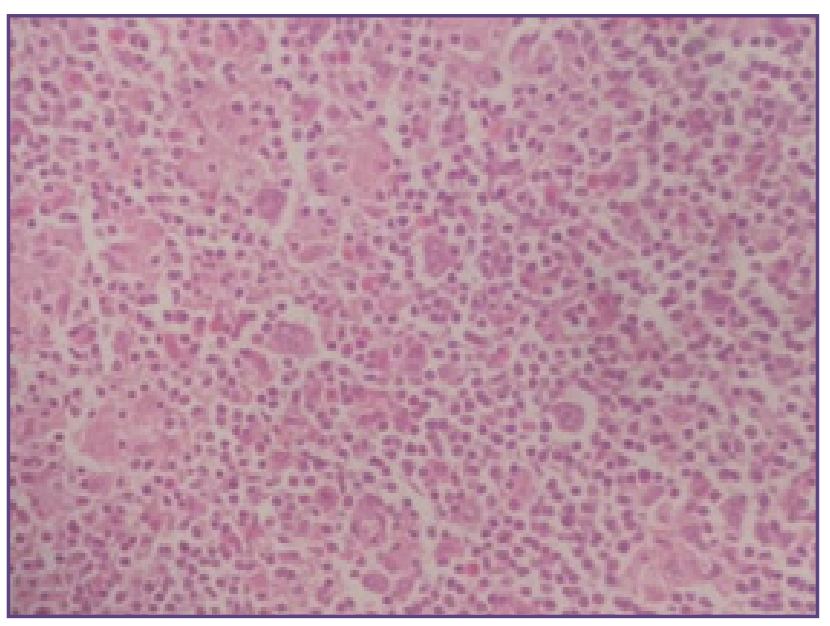

Fig. 4: Section showing many atypical mono-nuclear cells with few classical Reed Sternberg cells alongwith the population of polymorphus cells. (H\&E x 10X). 


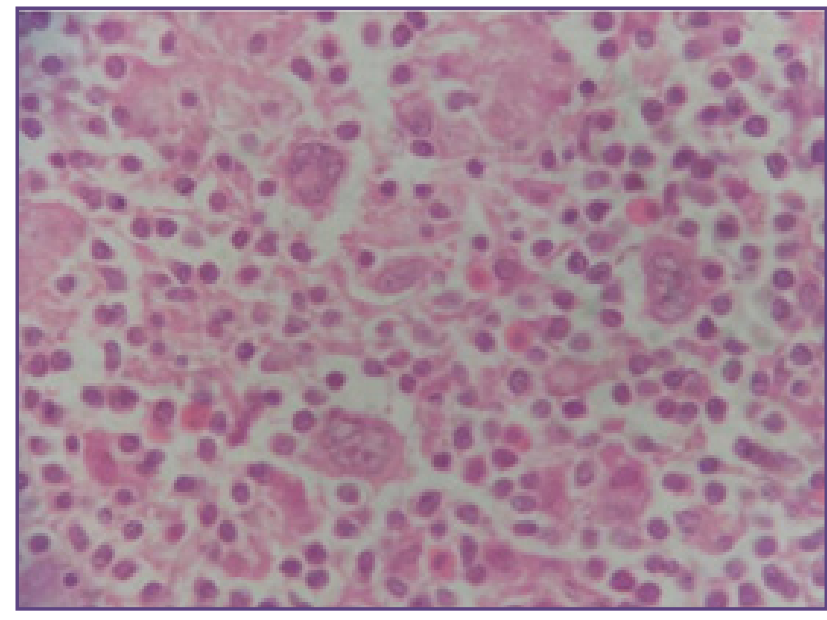

Fig. 5: Section showing atypical mono-nuclear cells, classical Reed Sternberg cells alongwith neutrophils, mature reactive lymphocytes, eosinophils \& plasma cells. (H\&E x 40X).

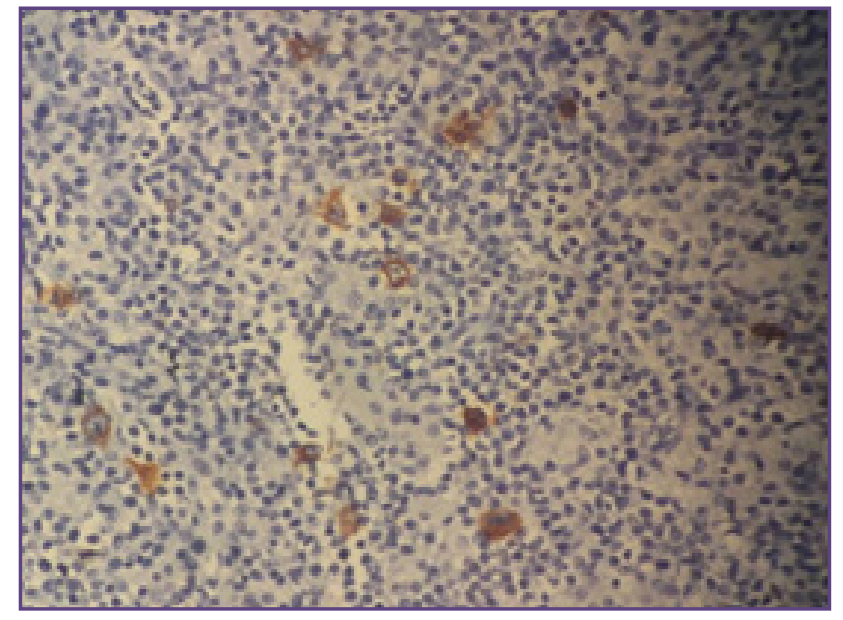

Fig. 6: Reed Sternberg cells express the dot like positivity of CD30 molecule. (IHC x 10X).

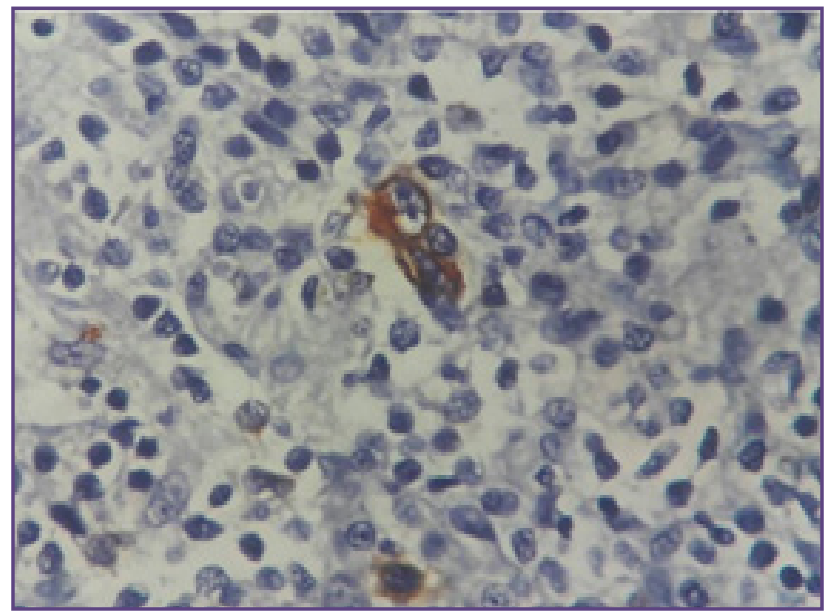

Fig. 7: Neoplastic cells show membrane bound and dot like CD30 positivity. (IHC x 40X).

\section{Discussion}

Lymphoma is the fourth most commonly diagnosed malignancy during pregnancy and nodular sclerosis HL is the most prevalent subtype. It has a reported incidence ranging from 1 per 1,000 to 1 per 6,000 deliveries. ${ }^{[2]}$ Since it is one of the most curable forms of lymphoma so FNAC plays a very important role in quick diagnosis and effective treatment of such cases. Radiological investigations have their limitations in pregnant females. CT of abdomen and pelvis could expose the fetus to radiation of at least 0.02 Gy, which is considered potentially teratogenic. Therefore, abdominal and pelvic CT should be avoided during pregnancy. PET/CT could be performed after delivery to assess treatment response. Staging of a pregnant patient with HL should be based on history, physical examination, routine blood tests, and bone marrow biopsies, as well as on MRI or ultrasonography. ${ }^{[3]}$

FNAC is a minimally invasive procedure which can be easily performed in pregnant females. Major procedures may lead to spontaneous labor. Hodgkin's lymphoma has typical cytological features. In the light of morphological, phenotypic, genotypic, and clinical findings, it is divided into two main types: lymphocyte predominant (LP-HL) and classical HL (CHL). CHL included the following subtypes: nodular sclerosis (NS-CHL), mixed cellularity (MC-CHL), lymphocyte depletion (LD-CHL), and the diffuse form of the lymphocyte rich CHL (LR-CHL). ${ }^{[4]} \mathrm{LP}-$ HL represents $4-5 \%$ of all HL cases, and differs greatly 
from the other type in terms of morphology, phenotype, genotype, and clinical behaviour. It has a unimodal age distribution, with a single peak in the 4th decade, which contrasts with the two peaks of CHL, one in the 3rd and the other in the 7th decade. The disease usually involves single cervical, axillary, or inguinal nodes rather than groups of nodes. Bone marrow involvement is rare. The tumour has a very indolent course, with prolonged disease free intervals with good prognosis. The various morphological and immuno-phenotypical features differentiating it from classical HL and T-cell rich B cell lymphoma are discussed in Table-1. ${ }^{[5,6]}$

FNAC not only provides early diagnosis but also helps in ancillary techniques like cell block preparation. With the help of cell block immuno-histochemical pattern could be studied without doing excision biopsy. Immunohistochemical markers provide prognostic stratification, and correlation with clinical features can outline the risk profile of the patients more accurately which may have a critical impact on therapeutic decisions. Therefore, immuno-histochemical evaluations should be considered in all cases. In difficult cases, flow cytometry can also be done on the cytology aspirates for immuno-phenotyping. In our case, the cell-block could not be processed as it was nodular sclerosis HL so cell yield was very less on aspiration. In all other types of HL or lymphomas the FNAC yield is good enough for the preparation of the cell block. So in our case the multi-disciplinary team decided to perform biopsy under local anesthesia. Furthermore, immuno-histochemistry plays a very important role in differentiating HL from anaplastic large cell lymphomas as discussed in Table-2. ${ }^{[5,7,8]}$

$\mathrm{HL}$ behaves similar in pregnant as well as non-pregnant women. The clinical behavior and histologic subtypes of HL in pregnancy are no different from those of non-pregnant women of similar age. The more frequent histologic type found in a study of 17 women diagnosed with $\mathrm{HL}$ at approximately 22 weeks of gestation was classic nodular sclerosing HL, found in 13 patients. It is very important to know about the course and progression of the disease during pregnancy because previously it was thought that pregnancy worsened the prognosis of HL. A case-controlled study of 48 cases of pregnancy-associated HL, however, showed a 20-year survival rate. No metastases to the placenta or the foetus were described. Although prognosis does not appear to be adversely affected, pregnancy imposes significant limitations on HL management. Exposure of the developing fetus to teratogens should be avoided whenever possible, but delaying treatment may be deleterious to the mother. When lymphoma is diagnosed during the second or third trimester, full-dose chemotherapy can be administered safely without any adverse effect on fetal outcome. Avoiding chemotherapy if possible during the first trimester of pregnancy is generally recommended. The risk of birth defects increases if exposure occurs in gestation weeks 5-12, when organogenesis takes place. Significant exposure to cytotoxic agents during the first 4 weeks of gestation may result in spontaneous abortion. Treatment within the first trimester of pregnancy remains controversial. The treatment approach should be individualized in accordance with the gestation period, disease stage and localization, and progression of symptoms and signs. A follow-up of high-risk pregnancies should be done with a specialized obstetrician. ${ }^{[9,10]}$

\section{Conclusion}

Though Hodgkin's lymphoma is a commonly known entity it is very important to know its course and progression in pregnant females. This case is presented to highlight the importance of FNAC in early diagnosis. Cell block preparation can help to study the immuno-histochemical pattern and characterization of the lesion thus helping the obstreticians in starting a definite therapy.

\section{References}

1. Pentheroudakis G, Pavlidis N. Cancer and pregnancy: poena magna, not anymore. Eur J Cancer. 2006;42(2):126-140.

2. Bachanova V, Connors JM. Hodgkin lymphoma in pregnancy. Curr Hematol Malig Rep. 2013 Sep;8(3):211-7.

3. Kassab C, Fleury G, Weinschenker P, Rodrigues F, Hamerschlak N. Hodgkin's disease and pregnancy: case series and proposal for treatment protocol. Einstein. 2011;9:216-219.

4. Harris NL, Jaffe ES, Diebold J, et al. The World Health Organization classification of neoplastic diseases of the haematopoietic and lymphoid tissues: report of the Clinical Advisory Committee Meeting, Airlie House, Virginia, November 1997. Histopathology 2000;36:69-87.

5. Sanchez M, Pellicer B, Cozar MP, Martínez-Sanjuan V, Villegas C, Carbonell F. Hodgkin Lymphoma in Pregnancy. Clinical Advances in Hematology \& Oncology2013; (11):533-36.

6. Mason DY, Banks PM, Chan J, et al. Nodular lymphocyte predominance Hodgkin's disease: a distinct clinicopathologic entity. Am J Surg Pathol 1994;18:526-30.

7. Falini B, Pileri S, Stein H, et al. Variable expression of leukocyte common antigen (CD45) in CD30 (Ki-1)-positive anaplastic large cell (ALC) lymphoma. Implication for the differential diagnosis between lymphoid and non-lymphoid malignancies. Hum Pathol 1990;21:624-9. 
8. Pileri SA, Ascani S, Leoncini L, Sabattini E, Zinzani P L, Piccaluga P P, et al. Hodgkin's lymphoma: the pathologist's viewpoint J Clin Pathol 2002;55:162-176.

9. Fadilah SA, Leong CF, Jamil MY, Cheong SK, Rozilaila R. Pregnancy complicated by Hodgkin's disease. Med J
Malaysia. 2006;61(3):358-360.

10. Eyre TA, Lau IJ, Mackillop L, Collins GP. Management and controversies of classical Hodgkin lymphoma in pregnancy. Br J Haematol. 2015 Jun;169(5):613-30.

*Corresponding author:

Dr. Pallavi Agrawal, C/o Dr. Rakesh Mehra, Mehra House, Opposite A. N. College, Rose Bud School Office Lane, Boring Road, Patna (Bihar) India PIN - 800013

Phone: +917739165410

Email: dr.pallavimame@gmail.com

Financial or other Competing Interests: None. 